A. M. ODLYZKO

\title{
Bounds for discriminants and related estimates for class numbers, regulators and zeros of zeta functions : a survey of recent results
}

Séminaire de Théorie des Nombres de Bordeaux, tome 2, nº 1 (1990), p. 119-141

<http://www.numdam.org/item?id=JTNB_1990_2_1_119_0>

(C) Université Bordeaux 1, 1990, tous droits réservés.

L'accès aux archives de la revue "Séminaire de Théorie des Nombres de Bordeaux » (http://jtnb.cedram.org/) implique l'accord avec les conditions générales d'utilisation (http://www.numdam.org/conditions). Toute utilisation commerciale ou impression systématique est constitutive d'une infraction pénale. Toute copie ou impression de ce fichier doit contenir la présente mention de copyright.

\section{Numdam}

Article numérisé dans le cadre du programme

Numérisation de documents anciens mathématiques

http://www.numdam.org/ 
Séminaire de Théorie des Nombres, Bordeaux 2 (1990), 119-141

\title{
Bounds for discriminants and related estimates for class numbers, regulators and zeros of zeta functions : a survey of recent results.
}

\author{
par A.M. ODLYZKO
}

\begin{abstract}
Résumé - Nous présentons une bibliographie d'articles récents sur les bornes inférieures des discriminants de corps de nombres et sur des sujets voisins. Nous discutons quelques unes des principales méthodes, et nous donnons les résultats principaux et des problèmes ouverts.
\end{abstract}

Abstract - A hibliography of recent papers on lower bounds for discriminants of number fields and related topics is presented. Some of the main methods, results, and open problems are discussed.

\section{Introduction.}

This paper presents a guide to the recent literature on lower bounds for discriminants of number fields and on several related topics, and discusses some open problems in these areas. Let $K$ be an algebraic number field of degree $n=n_{K}$ over the rationals $\mathbb{Q}$ with $r_{1}$ real and $2 r_{2}$ complex conjugate fields, so that $n=r_{1}+2 r_{2}$. Let $D=D_{K}$ denote the absolute value of the discriminant of $K$. (Recall that the sign of the discriminant is $(-1)^{r_{2}}$, so little information is lost by considering just $D$.) The root-discriminant $r d=r d_{K}$ of $K$ is defined by

$$
r d_{K}=D_{K}^{1 / n_{K}} .
$$

The Dedekind zeta function of $K$ is denoted by $\zeta_{K}(s)$. The Generalized Riemann Hypothesis (GRH) for $K$ is the conjecture that all the zeros of the zeta function $\zeta_{K}(s)$ that lie within the critical strip $0<\operatorname{Re}(s)<1$ actually lie on the critical line $\operatorname{Re}(s)=1 / 2$.

For $K=\mathbf{Q}, D=1$, and one of Minkowski's fundamental results was the proof that $D>1$ for $n>1$. He later obtained a lower bound for $D$ that was exponential in $n$. Minkowski's bound was subsequently improved by many authors. Most of the papers on this subject before 1970, including all those with the strongest estimates, used geometry of numbers methods.

Manuscrit reçu le 25 août 1989, révisé le 26 février 1990 
H. Stark [B1,B2] introduced a new analytic method for proving lower bounds for discriminants by showing that for every complex $s$ (other than 0,1 , or a zero of $\left.\zeta_{K}(s)\right)$,

$$
\begin{aligned}
\log D=r_{1}(\log \pi-\psi(s / 2))+ & 2 r_{2}(\log (2 \pi)-\psi(s)) \\
& -\frac{2}{s}-\frac{2}{s-1}+2 \sum_{\rho}^{\prime} \frac{1}{s-\rho}-2 \frac{\zeta_{K}^{\prime}}{\zeta_{K}}(s),
\end{aligned}
$$

where $\rho$ runs over the zeros of $\zeta_{K}(s)$ in the critical strip, and $\Sigma^{\prime}$ means that the $\rho$ and $\bar{\rho}$ terms are to be taken together, and

$$
\psi(s)=\frac{\Gamma^{\prime}}{\Gamma}(s) .
$$

This identity is a variant of the classical identity [I2; Satz 180] that comes from the Hadamard factorization of $\zeta_{K}(s)$, and Stark noticed that two of the constants that occur in that identity and which are hard to estimate can be eliminated. (It is ironic that Landau did not notice this, since he proved the corresponding result for Dirichlet $L$-functions [I1]. IIad he seen the extension to Dedekind zeta functions, a large part of the recent research might have been done 70 years earlier.) Since

$$
-\frac{\zeta_{K}^{\prime}}{\zeta_{K}}(s)=\sum_{\mathfrak{P}} \sum_{m=1}^{\infty} \frac{\log N \mathfrak{P}}{N \mathfrak{P}^{m s}}
$$

for $\operatorname{Re}(s)>1$, where $\mathfrak{P}$ runs over the prime ideals of $K$, one finds that for $s$ real, $s>1$,

$$
\log D \geq r_{1}(\log \pi-\psi(s / 2))+2 r_{2}(\log (2 \pi)-\psi(s))-2 / s-2 /(s-1)
$$

By taking $s=1+n^{-1 / 2}$, say, one obtains from (1.5) the estimate

$$
D \geq\left(4 \pi e^{C}\right)^{r 1}\left(2 \pi e^{C}\right)^{2 r_{2}} e^{o(n)} \text { as } n \rightarrow \infty
$$

where $C=0.5772156 \ldots$ denotes Euler's constant, and

$$
\begin{aligned}
4 \pi e^{C} & =22.3816 \ldots, \\
2 \pi e^{C} & =11.1908 \ldots .
\end{aligned}
$$

These estimates are substantially better than Minkowski's, although not as good as some of the more recent geometry of numbers bounds. 
The Stark bound (1.5) was improved by the author in a series of papers $[\mathrm{B} 3, \mathrm{~B} 4, \mathrm{~B} 5]$. Those papers used the fundamental identity (1.2) and combinations of identities derived from it by differentiation with respect to $s$. This led to substantial improvements on previous lower bounds for discriminants. Some of the bounds of those papers assume the GRH, and others are unconditional.

Serre [B7] introduced the use of the explicit formulas of Guinand [I5,I6] and Weil $[17, I 10]$ to discriminant bounds. This enabled him to improve the author's GRH bounds. What's perhaps most important, the resulting formulas provided a much more elegant approach to bounding discriminants, which made it clear what the requirements and limitations of the method are. Serre's approach was extended to provide unconditional bounds by the author, and further improvements were made by Poitou and the author [B9,B10]. The latest results in this area are summarized in Section 2.

Discriminant bounds have numerous applications, and there has been very substantial work in recent years on this and related topics. The purpose of this note is to present a guide to the recent literature and state some of the outstanding open problems. The references that are listed appear to be fairly complete regarding the main topic, but cover only some of the applications. The comments in this and the succeeding sections are much less complete, due to the extensive literature in this area. Several topics, such as determination of fields of small discriminants (see Section $F$ for references), the very interesting recent work on relative conductors [E13, E14, E15], and Mestre's work [H1] on conductors of elliptic curves are not dealt with in the text at all.

\section{Lower bounds for discriminants.}

In this section we state the latest bounds for discriminants obtained by use of explicit formulas of prime number theory, as well as some of their applications. We also discuss how close these bounds are to being optimal.

Consider a differentiable function $F: \mathbb{R} \rightarrow \mathbf{R}$, with $F(-x)=F(x)$, $F(0)=1$, and such that

$$
|F(x)|,\left|F^{\prime}(x)\right| \leq c e^{-(1 / 2+\epsilon)|x|} \text { as }|x|-\infty
$$

for some constants $c, \epsilon>0$. Define

$$
\Phi(s)=\int_{-\infty}^{\infty} F(x) e^{(s-1 / 2) x} d x .
$$


Then the explicit formula for the discriminant states that

$$
\begin{aligned}
\log D & =r_{1} \pi / 2+n(C+\log (8 \pi)) \\
& -n \int_{0}^{\infty} \frac{1-F(x)}{2 \sinh (x / 2)} d x-r_{1} \int_{0}^{\infty} \frac{1-F(x)}{2 \cosh (x / 2)} d x \\
& -4 \int_{0}^{\infty} F(x) \cosh (x / 2) d x+\sum_{\rho}^{\prime} \Phi(\rho) \\
& +2 \sum_{\mathfrak{P}} \sum_{m=1}^{\infty} \frac{\log N \mathfrak{P}}{N \mathfrak{P}^{m / 2}} F(m \log N \mathfrak{P}) .
\end{aligned}
$$

For a derivation of this formula, see [B9, B10]. The Guinand [I5, I6] and Weil [I7,I10] formulas are more general, although less explicit as to some terms. See Besenfelder [I11] for even more general kernels. Many of the published formulas, including those of Guinand, are derived only for the Riemann zeta function, but there is no difficulty in extending them to Dedekind zeta functions.

In the absence of any special knowledge about zeros and prime ideals (see sections 4 and 6 and later parts of this section for a discussion of their influence), in order to obtain a lower bound for $D$ from (2.3) one selects $F(x) \geq 0$ for all $x$ and $\operatorname{Re}(\Phi(s)) \geq 0$ for all $s$ in the critical strip, so that the contributions of the prime ideals and zeros are nonnegative. The above nonnegativity conditions on $F(x)$ and $\Phi(s)$ are equivalent to the requirement that

$$
F(x)=\frac{f(x)}{\cosh (x / 2)},
$$

where $f(x) \geq 0$ and $f(x)$ has nonnegative Fourier transform. The best currently known unconditional bounds are obtained by selecting $f(x)=$ $g(x / b)$ for some parameter $b$ (depending on $r_{1}$ and $r_{2}$ ), where $g(x)$ is a certain function constructed by L. Tartar [B10,B11]. With this choice one finds that

$$
\begin{aligned}
r d & =D^{1 / n} \geq\left(4 \pi e^{1+C}\right)^{r_{1} / n}\left(4 \pi e^{C}\right)^{2 r_{2} / n}-O\left(n^{-2 / 3}\right) \\
& =(60.8395 \ldots)^{r_{1} / n}(22.3816 \ldots)^{2 r_{2} / n}-O\left(n^{-2 / 3}\right) .
\end{aligned}
$$

No other choice of $f(x)$ can give a lower bound for $r d$ that has a larger main term than Eq. (2.5). Many choices of $g(x)$ other than Tartar's give 
the same estimates as Eq. (2.5), but with larger constants in the remainder term $O\left(n^{-2 / 3)}\right.$. The question of how small the remainder term can be made is still open. Tartar showed that his $g(x)$ was optimal in a certain class, but it is possible that better choices exist that do not satisfy his assumptions. Furthermore, it is not known whether the optimal functions $f(x)$ are of the form $g(x / b)$ for a fixed function $g(x)$.

Open Problem 2.1. What functions $f(x)$ satisfying $f(x) \geq 0$ and having nonnegative Fourier transforms give the best unconditional lower bounds for discriminants?

The bound (2.5) was stated above only in a very rough asymptotic form. There are much more explicit versions with precise estimates of the remainder term in [B10]. Also, there are extensive tables of bounds for all degrees $n \leq 100$ (and for some fields of higher degrees) in [B11]. Some of these tables are reprinted in [B12].

When one assumes the GRH for $\zeta_{K}(s)$, much better results are possible. In this case one only reeds $F(x) \geq 0$ such that the Fourier transform of $F(x)$ is nonnegative.

Open Problem 2.2. What functions $F(x)$ give the best GRH bounds for discriminants?

There are many choices of $G(x)$ such that $F(x)=G(x / b)$ for a proper choice of the scaling parameter $b$ gives the bound

$$
\begin{aligned}
r d & =D^{1 / n} \geq\left(8 \pi e^{C+\pi / 2}\right)^{r_{1} / n}\left(8 \pi e^{C}\right)^{2 r_{2} / n}-\mathrm{O}\left((\log n)^{-2}\right. \\
& =(215.3325 \ldots)^{r_{1} / n}(44.7632 \ldots)^{2 r_{2} / n}-\mathrm{O}\left((\log n)^{-2}\right) .
\end{aligned}
$$

Just as in the unconditional case, no choice of $F(x)$ can give a better main term. As was noted in [115], it is possible to show, using some results of Boas and Kac [14], that the choice of $G(x)$ proposed by the author (see [B10]) is asymptotically optimal among all possible functions in terms of minimizing the remainder term $O\left((\log n)^{-2}\right)$. However, it is not known whether the optimal $F(x)$ have to be of the form $F(x)=G(x / b)$ for a fixed function $G(x)$.

As in the case of unconditional bounds, there are estimates that are much more precise than (2.6). Fairly extensive tables of bounds for moderate degrees have been prepared [B8], and some of them have been reprinted in [B12].

Explicit formula bounds can also be obtained for conductors of Artin $L$ functions. (This was done in [B6] using logarithmic derivatives.) However, 
it is necessary to assume Artin's conjecture, as otherwise the poles of the $L$-functions might give a contribution that seems hard to control.

A point of view advocated by J.-P. Serre is to ask for lower bounds for discriminants of global fields $K$ whose completions at some places give a prescribed collection of local fields $K_{j}$. If the $K_{j}$ are the reals taken $r_{1}$ times and the complex numbers taken $2 r_{2}$ times, we are in the standard setting described above. If the $K_{j}$ are a certain number of copies of a function field over $F_{q}$, and one replaces the discriminant by the genus, one is led to estimate the minimal genus of a curve over $F_{q}$ that has at least a given number of points [I14].

One of the main applications of discriminant bounds is to estimate class numbers. Some of the results give lower bounds for class numbers of totally complex extensions of totally real fields, for example [B2,B3,D4], that grow very fast. This is in contrast to the typical situation, where it is expected on heuristic grounds that class numbers will equal 1 very often [I13,I17]. So far, though, we do not even know whether there exist infinitely many number fields of class number one.

Discriminant bounds are often used to show that class numbers of particular fields are small. The basic tool that is used is the Hilbert class field. If a field $K$ has class number $h$, then there is an extension $L$ of $K$ with $[L: K]=h$ such that $r d_{L}=r d_{K}$. If lower bounds for discriminants imply that $r d_{L}>r d_{K}$ for all fields $L$ with $[L: K] \geq 2$, then we can conclude that $L=K$ and $h=1$. Most applications (see Section D of References) are more sophisticated, and use discriminant bounds to get upper bounds for class numbers, which are then lowered by algebraic methods.

For some other applications of discriminant bounds, see [I12,I16].

For a long time it was conjectured that if $d_{n}$ denotes the minimal rootdiscriminant of a number field of degree $n$, then $d_{n} \rightarrow \infty$ as $n \rightarrow \infty$. (This is known to be true for abelian fields.) If true, this would have shown that all Hilbert class field towers terminate, and so all number fields could be embedded in fields of class number one. However, Golod and Shafarevich showed that infinite Hilbert class field towers do exist. The best current results are due to Martinet $[\mathrm{B} 12, \mathrm{C} 2]$ who showed that there is an infinite sequence of totally real number fiels (with degrees equal to powers of 2) with

$$
r d=4 \cdot(3 \cdot 5 \cdot 7 \cdot 23 \cdot 29)^{1 / 2}=1058.565 \ldots
$$

and an infinite sequence of totally complex number fields (this time with 
degrees 5 times powers of 2 ) with

$$
r d=11^{4 / 5} \cdot 2^{3 / 2} \cdot 23^{1 / 2}=92.368 \ldots
$$

It is thought likely that the field $Q\left((-3.5 .7 .13)^{1 / 2}\right)$ with

$$
r d=2 \cdot(3 \cdot 5 \cdot 7 \cdot 13)^{1 / 2}=73.891 \ldots
$$

also has an infinite class field tower, but this has not been proved.

Open problem 2.3. What are the minimal discriminants of number fields of various degrees?

Tables 1-4 present some data on this problem. It is interesting that the lower bounds (especially those that assume the GRH) are very close to the smallest values of root-discriminants that are known for totally complex fields. Even for totally real fields, the discrepancy is not large, except for $n=7$. The anomalous results for $n=7$ suggest strongly that the minimal root discriminants probably do not increase monotonically with the degree, especially if one restricts attention to totally real fields.

The GRH bounds presented in Tables 3 and 4 can be improved somewhat. For example, as was noted in [B8], from which these bounds are taken, one can show that all fields of degree 8 satisfy $r d \geq 5.743$, and not just $r d \geq 5.734$.

The entry for $n=9$ in Table 4 comes from the field generated by a root of the polynomial $x^{9}-2 x^{8}-7 x^{7}+14 x^{6}+15 x^{5}-30 x^{4}-10 x^{3}+19 x^{2}+2 x-1$, which was discovered recently by Leutbecher (unpublished). The discriminant of the polynomial (and thus of the field) is $9,685,993,193$, a prime, but it is not known yet whether it is minimal.

Known constructions for infinite Hilbert class field towers rely on working with the 2-part or 3-part of the class group, and so produce fields with degrees that are powers of a small number of primes. Essentially nothing is known about fields of prime degree, which leads to the following problem.

Open Problem 2.4. Is $d_{p}$ bounded as $p \rightarrow \infty$ with $p$ prime?

Sor far we have been discussing discriminants of number fields. IIowever, another relevant question, asked by J.-P. Serre and others, concerns discriminants of polynomials. Let $M_{n}$ denote the smallest absolute value of the discriminant of an irreducible monic polynomial with integral coefficients and degree $n$.

Open Problem 2.5. Is there an infinite subset $S$ of positive integers such that $M_{n}^{1 / n}$ is bounded for $n \in S$ ? 
Nothing is known on this topic. The analytic methods discussed in this paper do not apply to this situation. When one examines known fields with minimal discriminants, they tend to have algebraic integers $\alpha$ that generate them over $Q$ and such that the discriminant of the minimal polynomial of $\alpha$ equals the discriminant of the field. (This means that if $n$ is the degree of $\alpha$, then $1, \alpha, \alpha^{2}, \ldots, \alpha^{n-1}$ forms a basis of the algebraic integers of the field over the rational integers, or what is called a power basis.) However, it is generally thought that this phenomenon does not persist for higher degree fields. Already in the case of the field with $\left(n, r_{2}\right)=(8,2)$ that has the smallest known discriminant for all such fields, no power basis has been found so far.

\section{Elkies' GRH bound for discriminants}

Noam Elkies has observed that a form of the GRH bound (2.6) can be obtained without invoking the Guinand-Weil explicit formulas, by relying on the Landau-Stark formula (1.2) along the lines of the estimates of [B3, B4, B5]. The remainder term in Elkies' estimate appears to be much worse than in the explicit formula estimates, so it is not of practical significance, but it is interesting that this can be done at all. With Elkies' permission, we present a sketch of the proof here.

From (1.1), we see that $-\zeta_{K}^{\prime}(s) / \zeta_{K}(s)$ and its derivatives of even order with respect to $s$ are positive for $s>1$, and the derivatives of odd order negative; thus by differentiating (1.1) $m$ times $(m=0,1,2, \ldots)$ we find (with $\rho=1 / 2+i \gamma$ for real $\gamma$ under the assumption of the GRH)

$$
0>(-1)^{m}\left[r_{1} \frac{d^{m}}{d s^{m}}(\log \pi-\psi(s / 2))+2 r_{2} \frac{d^{m}}{d s^{m}}(\log 2 \pi-\psi(s))\right]
$$

$$
+m !\left[2 \operatorname{Re} \sum_{\rho}(s-(1 / 2+i \gamma))^{-m-1}-\frac{2}{(s-1)^{m+1}}+\frac{2}{s^{m+1}}\right] .
$$

Elkies' idea is that for fixed $s>1$ and large $m$ the term in $(s-1)^{-(m+1)}$ is negligible, and so by dividing the rest of $(3.1)$ by $2^{m} m$ ! and summing over $m$ we obtain (1.2) with $s$ replaced by $s-1 / 2$ (Taylor expansion about $s)$; since $\operatorname{Re}(1 /(s-1-i \gamma))$ is still positive, we then find by bringing $s$ arbitrarily close to 1 that

$$
\log \left|D_{K}\right|>r_{1}(\log \pi-\psi(1 / 4))+2 r_{2}(\log 2 \pi-\psi(1 / 2))-o(n),
$$


and thus obtain the bound from the known special values

$$
\psi(1 / 2)=-\log 4-C, \psi(1 / 4)=-\log 8-\pi / 2-C .
$$

To make this rigorous, we argue as follows: for any small $\epsilon>0$, take $s_{0}=1+\epsilon$, and pick an integer $M$ so large that (i) the values at $s=s_{0}-1 / 2$ of the $M$-th partial sums of the Taylor expansions of $\psi(s)$ and $\psi(s / 2)$ about $s=s_{0}$ are within $\epsilon$ of $\psi\left(s_{0}-1 / 2\right)$ and $\psi\left(s_{0} / 2-1 / 4\right)$ respectively (this is possible because both functions are analytic in a circle of radius $1>1 / 2$ about $\left.s_{0}\right)$; (ii) the value at $s=s_{0}-1 / 2$ of the $M$-th partial sum of the Taylor expansion of $\operatorname{Re}(1 /(s-1 / 2-i \gamma))$ about $s=s_{0}$ is positive for all $\gamma>0$ (note that since $\operatorname{Re}(1 /(s-1-i \gamma))=\epsilon /\left(\epsilon^{2}+\gamma^{2}\right)$, and the value of the $M$-th partial sum of the Taylor expansion differs from this by

$$
\operatorname{Re} \frac{1}{[1+2(\epsilon-i \gamma)]^{M}(\epsilon+i \gamma)}<<\left(1+\epsilon^{2}+\gamma^{2}\right)^{-M / 2},
$$

it's clear that the positive value $\epsilon /\left(\epsilon^{2}+\gamma^{2}\right)$ dominates the error $\left(1+\epsilon^{2}+\gamma^{2}\right)^{-M / 2}$ for all $\gamma$ once $M$ is sufficiently large). Now divide (3.1) by $2^{m} m$ !, sum from $m=0$ to $M-1$, and set $s=s_{0}$ to obtain

$$
\begin{aligned}
\log \left|D_{K}\right|>r_{1}\left(\log \pi-\psi\left(s_{0} / 2-1 / 4\right)-\epsilon\right)+ \\
\\
2 r_{2}\left(\log 2 \pi-\psi\left(s_{0}-1 / 2\right)-\epsilon\right)+O(1) ;
\end{aligned}
$$

since $\epsilon$ was arbitrarily small and $s_{0}$ arbitrarily close to 1 , we're done.

\section{Prime ideals of small norms}

It is not known how much of a contribution is made by prime ideals of small norm to the minimal discriminants when one applies the identy (2.3). (See Section 6 for furth r discussion and numerical evidence on this point.) In some situations some prime ideals of small norm are known, and this can be exploited by computing their contributions to the explicit formula.

The kernels $F(x)$ that are used to obtain the best known discriminant bounds in the absence of any knowledge about prime ideals have the property that $F(x)$ decreases very rapidly as $x \rightarrow \infty$, and in some cases they do not decrease monotonically. This often makes it difficult to employ them 
to take advantage of prime ideals whose norms are not too small. In such cases, the author's older bounds [B3,B4,B5] have occasionally been used. However, in most cases one can obtain stronger and more elegant bounds by using explicit formulas. For example (see [I18] for an application), if one takes $1<\omega<2$,

$$
F(x)=\frac{1}{\cosh (\omega-1 / 2) x},
$$

then one finds that $\operatorname{Re} \Phi(s) \geq 0$ for $0 \leq \operatorname{Re}(s) \leq 1$, and that

$$
\begin{aligned}
\Phi(0) & =2 \int_{0}^{x} F(x) \cos (x / 2) d x \\
& =\frac{\pi}{\omega-1 / 2} \frac{\cos \frac{\pi}{4 \omega-2}}{1+\cos \frac{\pi}{2 \omega-1}}=\frac{1}{\omega-1}+O(\omega-1) .
\end{aligned}
$$

On the other hand,

$$
2 \sum_{\mathfrak{P}} \sum_{m=1}^{\infty} \frac{\log N \mathfrak{P}}{N \mathfrak{P}^{m / 2}} F(m \log N \mathfrak{P}) \geq-\frac{8}{3} \frac{\zeta_{K}^{\prime}}{\zeta_{K}}(\omega)
$$

by a term-by-term comparison of the series, and so one finds, for example, that

$$
\log D>r_{1} \log 60.7+2 r_{2} \log 22.35-\frac{8}{3} \frac{\zeta_{K}^{\prime}}{\zeta_{K}}(\omega)-\frac{2}{\omega-1}
$$

for all $\omega \in(1,1+\delta)$ for some $\delta>0$ and $n \geq n_{0}$. With more effort one can obtain more precise estimates. Further, if there are no prime ideals of norm 2 , for example, the $8 / 3$ in (4.4) can be replaced by 3 , and so on. One can also devise other kernels $F(x)$ that will emphasize the contributions of particular ideals.

\section{Minkowski constants and regulators}

The analytic bounds for discriminants that were discussed above are substantially better than earlier ones that came from geometry of numbers. On the other hand, the analytic bounds have the disadvantage that they 
apply only to discriminants. Geometry of numbers bounds are derived from results that say, typically, that if $L_{1}(\mathbf{x}), \cdots, L_{n}(\mathbf{x})$ are linear forms in $x_{1}, \cdots, x_{n}$ with $\mathbf{x}=\left(x_{1}, \cdots, x_{n}\right)$, then there are integer values of the $x_{i}$, not all of them zero, such that the product $\prod L_{j}(\mathbf{x})$ is small. These bounds imply Minkowski-type estimates for norms of ideals; typically they state that each ideal class of $K$ contains an ideal $\mathfrak{A}$ of small norm, say with

$$
N \mathfrak{A} \leq\left(C_{1}-o(1)\right)^{-r_{1} / 2}\left(C_{2}-o(1)\right)^{-r_{2}} D^{1 / 2} \text { as } n \rightarrow \infty,
$$

where $C_{1}$ and $C_{2}$ are some constants independent of $K$. (We will refer to $C_{1}$ and $C_{2}$ as Minkowski constants.) Since $N \mathfrak{A} \geq 1$, Eq. (5.1) immediately implies a discriminant bound

$$
D \geq\left(C_{1}-o(1)\right)^{r_{1}}\left(C_{2}-o(1)\right)^{2 r_{2}} \text { as } n \rightarrow \infty .
$$

Bounds of the form (5.1) contain more information than (5.2), though, and are useful in other problems.

Zimmert [E4] has discovered an ingenious analytical method that uses zeta functions of ideal classes to obtain improved Minkowski constants. (See Oesterlé's paper [E6] for an elegant reformulation of the method.) In particular, he showed that (5.1) holds with

$$
\begin{aligned}
C_{1}=\exp (4 \log 2+2 C) & =50.7555 \ldots, \\
C_{2}=\exp (\log 2+\log \pi+2 C) & =19.9316 \ldots,
\end{aligned}
$$

which is considerably better than the known geometry of numbers bounds. Furthermore, Zimmert showed that for every class $\mathcal{R}$, there is always an ideal $\mathfrak{A}$ either in $\mathcal{R}$ or in $\mathcal{D} \mathcal{R}^{-1}$ (where $\mathcal{D}$ denotes the different of $K$ ) for which (5.1) holds with

$$
\begin{aligned}
& C_{1}=\exp (C+\log 4 \pi+1)=60.8395 \ldots \\
& C_{2}=\exp (C+\log 4 \pi)=22.3816 \ldots
\end{aligned}
$$

This last estimate implies the unconditional bound (2.5) for discriminants, although with a worse error term.

Open Problem 5.1. What are the best possible Minkowski constants?

It would be interesting to find out whether one can obtain estimates such as those of (5.4) that would hold for every ideal class. It would be very striking if one could prove results that would let one take $C_{1} \approx 215$, 
$C_{2} \approx 44$, since these would give bounds for discriminants that currently can be obtained only under the assumption of the GRH. There does not seem to be any clear way to bring the GRH into Zimmert's method, since he works with zeta functions of ideal classes, which do not have an Euler product, and for which the GRH is in general false.

Open Problem 5.2. Obtain improved bounds for minima of products of several linear forms by analytic methods.

The Zimmert bounds apply only to products of linear forms coming from an integral basis of a number field. It is not known whether these linear forms are extremal in the sense that their minimal nonzero values at integer points are the largest among all linear forms.

Zimmert has also found an analytic method for proving lower bounds for regulators of number fields [E4]. His methods show that the regulator $R$ of $K$ satisfies

$$
\begin{aligned}
R & \geq \exp \left(r_{1} \log 2+n C+o(n)\right) \\
& =\exp \left(r_{1} \cdot 0.693 \ldots+n \cdot 0.577 \ldots+o(n)\right) \text { as } n \rightarrow \infty,
\end{aligned}
$$

which significantly improves on the results of Remak [I3,I8, I9]. (Inequality (5.5) is stronger than the asymptotic result stated by Zimmert in [E4], but E. Friedman has pointed out that it follows easily from Satz 3 of [E4].) Friedman [E10] has found another, related method for obtaining analytic bounds for regulators that is very effective for small degrees, and has proved, for example, that the smallest regulator of any number field is 0.2052 . Very recently, Friedman and Skoruppa [E16] have found a generalization and a much clearer formulation of Zimmert's method for obtaining regulator bounds, which will hopefully lead to substantial improvements on (5.5).

Open Problem 5.3. What are the best possible lower bounds for the regulator $R$ of a number field as functions of $r_{1}$ and $r_{2}$ ? What are the best bounds in terms of $r_{1}, r_{2}$, and $D$ ?

E. Friedman has suggested that the following regulator bound might be valid:

$$
\begin{aligned}
R & \geq \exp \left(r_{1}(\pi-2 \log 2+2 \log \pi) / 4+n(C / 2+\log 2)+o(n)\right) \\
& =\exp \left(r_{1} \cdot 1.011 \ldots+n \cdot 0.981 \ldots+o(n)\right) \text { as } n \rightarrow \infty
\end{aligned}
$$




\section{Low zeros of Dedekind zeta functions}

The Landau-Stark formula (1.2) as well as the Guinand-Weil explicit formula (2.6) are identities, and so when $D$ is larger than the bound we obtain for it by the method sketched earlier, this must be due either to the contribution of prime ideals or of zeros of $\zeta_{K}(s)$. The kernels used in the explicit formula bounds are such that the contribution of prime ideals of large norm is negligible, as is the contribution of zeros of $\zeta_{K}(s)$ that are far from the real axis. Therefore it is primarily the prime ideals of small norms and the low zeros that determine the sizes of the minimal discriminants. If we fix the degree and let the discriminant grow, then we can select a kernel $F(x)$ with bounded support, so that the contribution of the prime ideals will be bounded, and so it will be the zeros that will dominate. The interesting question is to ask what happens when we choose the optimal kernel $F(x)$ without any knowledge of zeros or prime ideals, and then ask which contribution is larger for the small discriminants.

Currently no methods are known for efficiently computing high zeros of Dedekind zeta functions of general nonabelian fields. For low zeros there is a very nice method of Friedman [H3]. However, this method has not been implemented yet, and in any case it requires one to compute small norms of ideals. Therefore it seemed much easier to compute the small norms of prime ideals (by factoring the minimal polynomial of a generator modulo rational primes), evaluate their contribution to the explicit formula (2.3), and obtain the contribution of the zeros by subtraction.

Table 5 presents the results of the computation that was carried out for six fields, those with the smallest discriminants for $\left(n, r_{2}\right)=(7,0),(8,0)$, $(8,4)$, and the next smallest for $\left(n, r_{2}\right)=(8,0)$ and $(8,4)$ and the one with the smallest known discriminant for $\left(n, r_{2}\right)=(9,0)$. In each case the kernel $F(x)$ that gives the GRH bounds of Tables 3 and 4 was used, so that in the notation of [B8], $b=1.9$ for $\left(n, r_{2}\right)=(7,0), b=1.6$ for $\left(n, r_{2}\right)=(8,4), b=2.05$ for $\left(n, r_{2}\right)=(8,0)$, and $b=2.2$ for $\left(n, r_{2}\right)=(9,0)$. The column labelled "deficiency" denotes the difference between $n^{-1} \log r d$ for each field and the GRH lower bound. The "ideals" column denotes the value of the sum over prime ideals in (2.3), and the "zeros" column the value of the sum over the zeros (obtained by subtracting the "ideals" column from the "deficiency" column). Finally, the "norms" column gives the norms of prime ideals that contributed to the sum.

Open Problem 6.1. What are the relative contributions of prime ideals and zeros to the explicit formula for minimal discriminants?

Table 5 suggests that these contributions are of comparable magnitude, 
but it would be nice to obtain data for higher degree fields. (This should not be too difficult, since the fields of small discriminant found by Martinet in [C3] are given quite explicitly as ray class fields.) It is worth noting that if we use the kernel $F(x)$ that gives the best unconditional bound, then the contribution of zeros becomes considerably larger relative to that of the prime ideals.

Open Problem 6.2. Do the zeros of $\zeta_{K}(s)$ in the critical strip approach the real axis as $n \rightarrow \infty$, and if they do, how fast do they do so, and how many of them are there?

There does not seem to be any hope of proving algebraically that fields with small discriminant must have prime ideals of small norm. If we fix the degree $n$, then we can find fields of that degree in which the smallest norm of a prime ideal will be $2^{n}$, although this usually seems to require a large discriminant. On the other hand, if we let $D \rightarrow \infty$ while keeping $n$ fixed, then one can show that there will be zeros of $\zeta_{K}(s)$ arbitrarily close to the real axis (roughly $c(\delta) \log D$ zeros in $0 \leq \operatorname{Im}(s)<\delta$ for some $c(\delta)>0$ ). One might hope that one could prove that there are many zeros near the real axis even for minimal discriminants. If one could obtain enough such zeros, one could prove improved discriminant bounds. Unfortunately the known bounds are far too weak for this. The best results appear to be due to.the author [G5], and show that on the GRH, $\zeta_{K}(s)$ has a zero on the critical line at height $O\left((\log n)^{-1}\right)$ as $n \rightarrow \infty$. Unconditionally, it has only been shown [G5] that there is a zero at height $\leq 0.54+o(1)$ as $n \rightarrow \infty$, and that for every $K$ with $n \geq 2$, there is a zero at height $<14$. (The first zero of the Riemann zeta function is at height $14.1347 \ldots$,.., so this result shows that the zeta function is extremal in terms of having its lowest zero as high as possible.)

Open Problem 6.3. Are the GRH bounds for discriminants valid even without the assumption of the GRH?

The unconditional bounds are weaker than the GRH ones because of the requirement that $\operatorname{Re} \Phi(s) \geq 0$ throughout the critical strip. However, if we consider any one of the kernels used in obtaining the GRH bounds (which are required only to satisfy $\operatorname{Re} \Phi(s) \geq 0$ on the critical line $\operatorname{Re} s=1 / 2$ ), they will usually have $\operatorname{Re} \Phi(s) \geq 0$ in large sectors of the critical strip. In particular, all these kernels are $\geq 0$ for $s$ real, so if the only violations of the GRH were on the real axis, the GRH bounds for discriminants would be valid ! As an illustration, consider the kernel $\Phi(s)=2(\pi b)^{1 / 2} \exp (b(s-$ $1 / 2)^{2}$ ) that was first suggested by Serre. For this kernel, $\operatorname{Re} \Phi(s) \geq 0$ for $s$ 
real, as well as for many other regions of the critical strip. In $|\operatorname{Im}(s)|>|\operatorname{Re}(s-1 / 2)|, \Phi(s)$ is very small, $(b$ is taken large for large $n)$, so by taking a linear combination $\Phi_{1}(s)=(1-\delta) \Phi(s)+\delta \Phi^{*}(s)$, where $\Phi^{*}(s)$ is a carefully chosen kernel of the type used for the unconditional bounds, and $\delta>0$ is small, we can ensure that $\operatorname{Re} \Phi_{1}(s) \geq 0$ in $|\operatorname{Im}(s)|>$ $|\operatorname{Re}(s-1 / 2)|+1 / 100$ for large $b$, say. The discriminant bound given by $\Phi_{1}(s)$ would be only slightly inferior to that of $\Phi(s)$. It would take a very unusual combination of zeros violating the GRH to make the sum over the zeros be very negative for all possible choices of $b$. Unfortunately, as far as we know, such unusual distributions might occur.

Acknowledgements. The author thanks A.-M. Bergé, E. Friedman, J. Martinet, and J.-P. Serre for their detailed comments on an earlier version of this manuscript.

Table 1. Minimal absolute values of discriminants of number fields of degree $n$ with $2 r_{2}$ complex conjugate fields.

$\begin{array}{lrrrrl}n & r_{2}=0 & r_{2}=1 & r_{2}=2 & r_{2}=3 & r_{2}=4 \\ 1 & 1 & & & & \\ 2 & 5 & 3 & & & \\ 3 & 49 & 23 & & & \\ 4 & 725 & 275 & 117 & & \\ 5 & 14,641 & 4,511 & 1,609 & & \\ 6 & 300,125 & 92,779 & 28,037 & 9,747 & \\ 7 & 20,134,393 & 2,306,599 & 612,233 & 184,607 & \\ 8 & 282,300,416 & ? & ? & ? & 1,257,728\end{array}$

Table 2. Minimal root-discriminants of number fields of degree $n$ with $2 r_{2}$ complex conjugate fields.

$\begin{array}{llllll}n & r_{2}=0 & r_{2}=1 & r_{2}=2 & r_{2}=3 & r_{2}=4 \\ 1 & 1 & & & & \\ 2 & 2.236 & 1.732 & & & \\ 3 & 3.659 & 2.844 & & & \\ 4 & 5.189 & 4.072 & 3.289 & & \\ 5 & 6.809 & 5.381 & 4.378 & & \\ 6 & 8.132 & 6.728 & 5.512 & 4.622 & \\ 7 & 11.051 & 8.110 & 6.710 & 5.653 & \\ 8 & 11.385 & ? & ? & ? & 5.787\end{array}$


Table 3. Small root-discriminants of totally complex fields and the best known lower bounds. (For $n \leq 8$, the root-discriminants are known to be minimal for each degree.)

$\begin{array}{lccc}n & D^{1 / n} & \begin{array}{c}\text { GRH } \\ \text { bound }\end{array} & \begin{array}{c}\text { unconditional } \\ \text { bound }\end{array} \\ 2 & 1.732 & 1.722 & 1.722 \\ 4 & 3.289 & 3.263 & 3.254 \\ 6 & 4.622 & 4.592 & 4.557 \\ 8 & 5.787 & 5.734 & 5.659 \\ 10 & 6.793 & 6.726 & 6.600 \\ 14 & 8.426 & 8.371 & 8.122 \\ 20 & 10.438 & 10.270 & 9.805 \\ 32 & 13.181 & 12.912 & 12.002 \\ 48 & 15.472 & 15.225 & 13.772\end{array}$

Table 4. Small root-discriminants of totally real fields and the best known lower bounds. (For $n \leq 8$, the root-discriminants are known to be minimal for each degree.)

$\begin{array}{clcc}n & D^{1 / n} & \begin{array}{l}\text { GRH } \\ \text { bound }\end{array} & \begin{array}{l}\text { unconditional } \\ \text { bound }\end{array} \\ 1 & 1 & 0.997 & 0.997 \\ 2 & 2.236 & 2.225 & 2.223 \\ 3 & 3.659 & 3.630 & 3.610 \\ 4 & 5.189 & 5.124 & 5.067 \\ 5 & 6.809 & 6.640 & 6.523 \\ 6 & 8.182 & 8.143 & 7.941 \\ 7 & 11.051 & 9.611 & 9.301 \\ 8 & 11.385 & 11.036 & 10.596 \\ 9 & 12.869 & 12.410 & 11.823\end{array}$

Table 5. Contributions of prime ideals and zeros to discriminant bounds for some fields. (See Section 6 for detailed explanation.)

$\begin{array}{lllllll}D & n & r_{2} & \text { deficiency } & \text { ideals } & \text { zeros } & \text { norms } \\ 20134393 & 7 & 0 & 0.1397 & 0.0761 & 0.0636 & 7,17,23,37,43 \\ 1257728 & 8 & 4 & 0.0092 & 0.0013 & 0.0079 & 16 \\ 1265625 & 8 & 4 & 0.0100 & 0.0026 & 0.0074 & 16,16 \\ 282300416 & 8 & 0 & 0.0311 & 0.0183 & 0.0128 & 16,41,47,47,47,47,49,49 \\ 309593125 & 8 & 0 & 0.0427 & 0.0029 & 0.0398 & 3,41,41 \\ 9685993193 & 9 & 0 & 0.0364 & 0.0143 & 0.0221 & 27,31,41,43,67,67,73,73,79,79\end{array}$




\section{REFERENCES}

References are grouped by topic, and are arranged approximately chronologically within each group. For most areas only papers published after 1970 are listed. References to earlier ones can be found in the publications listed here, especially in the book of Narkiewicz [A1].

\section{$A$ - Books and surveys}

1. W. NARKIEWICZ, Elementary and Analytic Theory of Algebraic Numbers, PWNPolish Scientific Publishers Warsaw (1974). MR 50\# 268. (Very complete references for work before 1973.)

2. L. C. WASHINGTON, Introduction to Cyclotomic Fields, Springer. 1982. MR 85g : 11001.

3. J. MARTINET, Méthodes géométriques dans la recherche des petits discriminants. pp. 147-179, Séminaire Théorie des Nombres, Paris, 1983-84, C. Goldstein éd., Birkhäuser Boston, 1985. MR 88h:11083.

\section{B - Analytic lower bounds for discriminants}

1. H.M. STARK, Some effective cases of the Brauer-Siegel theorem, Invent. math. 23 (1974), 135-152. MR 49 \# 7218.

2. H.M. STARK, The analytic theory of algebraic numbers, Bull. Am. Math. Soc. 81 (1975), 961-972. MR 56 \# 2961.

3. A.M. ODLYZKO, Some analytic estimates of class numbers and discriminants, Invent. math. 29 (1975), 275-286. MR 51 \# 12788.

4. A.M. ODLYZKO, Lower bounds. for discriminants of number fields, Acta Arith. 29 (1976), 275-297. MR 53 \# 5531.

5. A.M. ODLYZKO, Lower bounds for discriminants of number fields. II. MR 56 \# 309.

6. A.M. ODLYZKO, On conductors and discriminants. pp. 377-407, Algebraic Number Fields, (Proc. 1975 Durham Symp.), A. Fröhlich, ed., Academic Press 1977. MR 56 \#11961.

7. J.-P. SERRE, Minorations de discriminants. note of October 1975, published on pp. 240-243 in vol. 3 of Jean-Pierre SERRE, Collected Papers, Springer 1986.

8. A.M. ODLYZKO, Discriminant bounds. tables dated Nov. 29, 1976 (unpublished). Some of these bounds are included in Ref. B12.

9. G. POITOU, Minorations de discriminants (d'après A.M. Odlyzko), Séminaire Bourbaki. Vol. 1975/76 28ème année, Exp. No. 479, pp. 136-153, Lecture Notes in Math. \#567, Springer 1977. MR 55 \#7995.

10. G. POITOU, Sur les petits discriminants, Séminaire Delange-Pisot-Poitou. $18 \mathrm{e}$ année : (1976/77), Théorie des nombres, Fasc. 1, Exp. No. 6, 18pp., Secrétariat 
Math., Paris, 1977. MR 81i:12007.

11. F. DIAZ Y DIAZ, Tables minorant la racine n-ième du discriminant d'un corps de degré $n$. Publications Mathématiques d'Orsay 80.06. Université de Paris-Sud, Département de Mathématique, Orsay, (1980). 59 pp. MR 82i:12007. (Some of these bounds are included in Ref. B12.)

12. J. MARTINET, Petits discriminants des corps de nombres. pp. 151-193, Journées Arithmétiques 1980, J.V. Armitage, ed., Cambridge Univ. Press 1982. MR 84g:12009.

See also A3,D9,E4,E6,E10,E16.

\section{Constructions of flelds with small discriminants}

1. H.W. LENSTRA, Euclidean number fields of large degree, Invent. math. 38 (1977), 237-254. MR 55 \# 2836.

2. J. MARTINET, Tours de corps de classes et estimations de discriminants, Invent. math. 44 (1978), 65-73.. MR 57 \# 275.

3. J. MARTINET, Petits discriminants, Ann. Inst. Fourier (Grenoble) 29, no 1 (1979), 159-170. MR 81h:12006.

4. R. SCHOOF, Infinite class field towers of quadratic fields, J. reine angew. Math. 372 (1986), 209-220. MR 88a:11121.

See also A3,B12.

\section{Bounds for class numbers}

1. J.M. MASLEY, Odlyzko bounds and class number problems. pp. 465-474, Algebraic Number Fields (Proc. Durham Symp., 1975), A. Fröhlich, ed., Academic Press 1977. MR 56 \# 5493.

2. J.M. MASLEY, Class numbers of real cyclic number fields with small conductor, Compositio Math. 37 (1978), 297-319. MR 80e:12005.

3. J.M. MASLEY, Where are number fields with small class numbers?. pp. 221-242, Number Theory, Carl ondale 1979, Lecture Notes in Math. \#751, Springer, 1979. MR 81f:12004.

4. J. HOFFSTEIN, Some analytic bounds for zeta functions and class numbers, Invent. math. 55 (1979), 37-47. MR 80k:12019.

5. J.M. MASLEY, Class groups of abelian number fields. pp. 475-497, Proc. Queen's Number Theory Conf. 1979, P. Ribenboim ed., Queen's Papers in Pure and Applied Mathematics no. 54, Queen's Univ., 1980, MR 83f:12007.

6. J. MARTINET, Sur la constante de Lenstra des corps de nombres, Sém. Theorie des Nombres de Bordeaux (1979-1980). Exp. \#17, 21 pp., Univ. Bordeaux 1980. MR 83b:12007.

7. F.J. van der LINDEN, Class number computations of real abelian number fields, Math. Comp. 39 (1982), 693-707. MR 84e:12005. 
8. A. LEUTBECHER and J. MARTINET, Lenstra's constant and Euclidean number fields, Astérisque 94 (1982), 87-131. MR 85b:11090.

9. A. LEUTBECHER, Euclidean fields having a large Lenstra constant, Ann. Inst. Fourier (Grenoble) 35. no.2 (1985), 83-106. MR 86j:11107.

10. J. HOFFSTEIN and N. JOCHNOWITZ, On Artin's conjecture and the class number of certain CM fields, Duke Math. J. 59 (1989), 553-563.

11. J. HOFFSTEIN and N. JOCHNOWITZ, On Artin's conjecture and the class number of certain CM fields-II, Duke Math. J. 59 (1989), 565-584.

See also B1,B2,B5.

E. Bounds for regulators and norms of ideals in ideal classes

1. M. POHST, Regulatorabschätzungen für total reelle algebraișche Zahlkörper, J. Number Theory 9 (1977), 459-492. MR 57 \# 268.

2. G. GRAS and M.-N. GRAS, Calcul du nombre de classes et des unités des extensions abéliennes réelles de $Q$, Bull. Sci. Math. 101 (2) (1977), 97-129. MR 58 \# 586.

3. M. POHST, Eine Regulatorabschätzung, Abh. Math. Sem. Univ. Hamburg 47 (1978), 95-106. MR 58 \# 16596.

4. R. ZIMMERT, Ideale kleiner Norm in Idealklassen und eine Regulatorabschätzung, Invent. math. 62 (1981), 367-380. MR 83g:12008.

5. G. POITOU, Le théorème des classes jumelles de R. Zimmert, Sém. de Théorie des Nombres de Bordeaux (1983-1984). Exp. \# 5, 4 pp., Univ. Bordeaux 1984, (Listed in MR 86b:11003.)

6. J. OESTERLÉ, Le théorème des classes jumelles de Zimmert et les formules explicites de Weil. pp. 181-197, Sém. Théorie des Nombres, Paris 1983-84, C. Goldstein ed., Birkhäuser Boston, 1985.

7. J. SILVERMAN, An inequality connecting the regulator and the discriminant of a number field, J. Number Theory 19 (1984), 437-442. MR 86c:11094.

8. T.W. CUSICK, Lower bounds for regulators. pp. 63-73 in Number Theory, Noordwijkerhout 1983, H. Jager ed., Lecture Notes in Math. \# 1068, Springer 1984. MR $85 \mathrm{k}: 11052$.

9. A.-M. BERGÉ and J. MARTINET, Sur les minorations géométriques des régulateurs. pp. 23-50, Séminaire Théorie des Nombres, Paris 1987-88, C. Goldstein ed., Birkhäuser Boston, 1990.

10. E. FRIEDMAN, Analytic formulas for regulators of number fields, Invent. math. 98 (1989), 599-622.

11. M. POHST and H. ZASSENHAUS, Algorithmic Algebraic Number Theory, Cambridge Univ. Press. (1989).

12. R. SCHOOF and L.C. WASHINGTON, Quintic polynomials and real cyclotomic fields with large class numbers, Math. Comp. 50 (1988), 543-556.

13. A.-M. BERGÉ and J. MARTINET, Notions relatives de régulateurs et de hauteurs, 
Acta Arith. 54 (1989), 155-170.

14. A.-M. BERGÉ and J. MARTINET, Minorations de hauteurs et petits régulateurs relatifs, Sém. Théorie des Nombres Bordeaux (1987-88). Exp.\#11, Univ. Bordeaux 1988.

15. A. COSTA and E. FRIEDMAN, Ratios of regulators in totally real extensions of number fields. to be published.

16. E. FRIEDMAN and N.-P. SKORUPPA, Explicit formulas for regulators and ratios of regulators of number fields. manuscript in preparation.

17. T. W. CUSICK, The regulator spectrum of totally real cubic fields. to appear.

\section{F. Determination of minimal discriminants}

1. P. CARTIER and Y. ROY, On the enumeration of quintic fields with small discriminants, J. reine angew. Math 268/269 (1974), 213-215. MR 50 \# 2119.

2. M. POHST, Berechnung kleiner Diskriminanten total reeller algebraischer Zahlkörper, J. reine angew. Math. 278/279 (1975), 278-300. MR 52 \# 8085.

3. M. POHST, The minimum discriminant of seventh degree totally real algebraic number fields. pp. 235-240, Number theory and algebra, H. Zassenhaus ed., Academic Press 1977. MR 57 \# 5952.

4. J. LIANG and H. ZASSENHAUS, The minimum discriminant of sixth degree totally complex algebraic number field, J. Number Theory 9 (1977), 16-35. MR 55 \# 305.

5. M. POHST, On the computation of number fields of small discriminants including the minimum discriminants of sixth degree fields, J. Number Theory 14 (1982), 99-117. MR 83g:12009.

6. M. POHST, P. WEILER, and H. ZASSENHAUS, On effective computation of fundamental units, Math. Comp. 38 (1982), 293-329. MR 83e:12005b.

7. D.G. RISH, On algebraic number fields of degree five, Vestnik Moskov. Univ. Ser. I Mat. Mekh. no 2, (1982), 76-80. English translation in Moscow Univ. Math. Bull. 37 (1982), no. 99-103. MR 83g:12006.

8.. F. DIAZ Y DIAZ, Valeurs minima du discriminant des corps de degré 7 ayant une seule place réelle, C.R. Acad. Sc. Paris 296 (1983), 137-139. MR 84i:12004.

9. F. DIAZ Y DIAZ, Valeurs minima du discriminant pour certains types de corps de degré 7, Ann. Inst. Fourier (Grenoble) 34, no 3 (1984), 29-38. MR 86d:11091.

10. K. TAKEUCHI, Totally real algebraic number fields of degree 5 and 6 with small discriminant, Saitama Math. J. 2 (1984), 21-32. MR 86i:11060.

11. H.J. GODWIN, On quartic fields of signature one with small discriminant. II, Math. Comp. 42 (1984). 707-711. Corrigendum, Math. Comp. 43 (1984), 621. MR 85i:11092a, 11092b.

12. F. DIAZ Y DIAZ, Petits discriminants des corps de nombres totalement imaginaires de degré 8, J. Number Theory 25 (1987), 34-52.

13. S.-H. KWON and J. MARTINET, Sur les corps résolubles de degré premier, 
J. reine angew. Math. 375/376 (1987), 12-23. MR 88g:11080.

14. F. DIAZ Y DIAZ, Discriminants minima et petits discriminants des corps de nombres de degré 7 avec cinq places réelles, J. London Math. Soc. 38 (2) (1988), 33-46.

15. J. BUCHMANN and D. FORD, On the computation of totally real quartic fields of small discriminant, Math. Comp. 52 (1989), 161-174.

16. S.-H. KWON, Sur les discriminants minimaux des corps quaternioniens. preprint 1987.

17. P. LLORENTE and J. QUER, On totally real cubic fields with discriminant $D<10^{7}$, Math. Comp. 50 (1988), 581-594.

18. J. BUCHMANN, M. POHST and J. v. SCHMETTOW, On the computation of unit groups and class groups of totally real quartic fields, Math. Comp. 53 (1989), 387-397.

19. A.-M. BERGÉ, J. MARTINET and M. OLIVIER, The computation of sextic fields with a quadratic subfield. in Math. Comp. to appear.

20. F. DIAZ Y DIAZ, Table de corps quintiques totalement réels. Université de Paris-Sud, Département de Mathématiques, Orsay, Report no. 89-14, 35 pp., 1989.

21. M. POHST, J. MARTINET and F. DIAZ Y DIAZ, The minimum discriminant of totally real octic fields, J. Number Theory. to appear.

\section{G. Small zeros of Dedekind zeta functions}

1. J. HOFFSTEIN, Some results related to minimal discriminants. pp. 185-194, Number Theory, Carbondale 1979, Lecture Notes in Math. \# 751, Springer 1979, MR 81d:12005.

2. A. NEUGEBAUER, On zeros of zeta functions in low rectangles in the critical strip. (in Polish), Ph.D. Thesis, A. Mickiewicz University, Poznan, Poland, 1985.

3. A. NEUGEBAUER, On the zeros of the Dedekind zeta-function near the real axis, Funct. Approx. Comment. Math. 16 (1988), 165-167.

4. A. NEUGEBAUER, Every Dedekind zeta-function has a zero in the rectangle $1 / 2 \leq \sigma \leq 1,0<t<60$, Discuss. Math.. to appear.

5. A.M. ODLYZKO, Lou zeros of Dedekind zeta function. manuscript in preparation.

\section{H. Other related papers}

1. J.-F. MESTRE, Formules explicites et minorations de conducteurs de variétés algébriques, Compositio Math. 58 (1986), 209-232. MR 87j:11059.

2. E. FRIEDMAN, The zero near 1 of an ideal class zeta function, J. London Math. Soc. 35 (2) (1987). 1-17. MR 88g:11087.

3. E. FRIEDMAN, Hecke's integral formula, Sém. Théorie des Nombres de Bordeaux (1987-88). Exp. \#5, 23 pp., Univ. Bordeaux 1988. 
I. Other papers cited in the text

1. E. LANDAU, Zur Theorie der Heckeschen Zetafunktionen, welche komplexen Charakteren entsprechen, Math. Zeit. 4 (1919), 152-162.. Reprinted on pp. 176-186 of vol. 7, Edmund Landau : Collected Works, P.T. Bateman, et al., eds., Thales Verlag.

2. E. LANDAU, Einführung in die elementare und analytische Theorie der algebraischen Zahlen und der Ideale, 2nd ed., Göttingen (1927). Reprinted by Chelsea, 1949.

3. R. REMAK, Über die Abschätzung des absoluten Betrages des Regulators eines algebraischen Zahlkörpers nach unten, J. reine angew. Math. 167. (1932), 360-378.

4. R.P. BOAS and M. KAC, Inequalities for Fourier transforms of positive functions, Duke Math. J. 12 (1945), 189-206. MR 6-265.

5.. A.P. GUINAND, $A$ summation formula in the theory of prime numbers, Proc. London Math. Soc. 50 (2) (1948), 107-119. MR 10, 104g.

6. A.P. GUINAND, Fourier reciprocities and the Riemann zeta-function, Proc. London Math. Soc. 51 (2) (1949), 401-414. MR 11, 162d.

7. A. WEIL, Sur les "formules explicites" de la théorie des nombres premiers, Comm. Sem. Math. Univ. Lund, tome supplémentaire (1952), 252-265. MR 14, 727e.

8. R. REMAK, Über Grössenbeziehungen zwischen Diskriminante und Regulator eines algebraischen Zahlkörpers, Compos. Math. 10 (1952), 245-285. MR 14, 952d.

9. R. REMAK, Über algebraische Zahlkörper mit schwachem Einheitsdefekt, Compos. Math. 12 (1954), 35-80. MR 16, 116a.

10. A. WEIL, Sur les formules explicites de la théorie des nombres, Izv. Akad. Nauk SSSR Ser. Mat. 36 (1972). 3-18. MR 52 \# 345. Reprinted in A. Weil, Oeuvres Scientifiques, vol. 3, pp. 249-264, Springer 1979.

11. H.-J. BESENFELDER, Die Weilsche "Explizite Formel" und temperierte Distributionen, J. reine angew. Math. 293/294 (1977), 228-257. MR 57 \# 254.

12. J.-P. SERRE. note on p. 710 in vol. 3 of Jean-Pierre SERRE, Collected Papers, Springer 1986.

13. H. COHEN and H.W. LENSTRA Jr., Heuristics on class groups of number fields. pp. 33-62 in Number Theory, Noordwijkerhout 1983, H. Jager ed., Lecture Notes in Math. \# 1068, Springer 1984. MR 85j:11144.

14. J.-P. SERRE, Sur le nombre des points rationnels d'une courbe algébrique sur un corps fini, C.R. Acad. Sci. Paris 296 (1983). sér. I, 397-402. MR 85b:14027. Reprinted on pp. 658-663 in vol. 3 of Jean-Pierre Serre, Collected Papers, Springer 1986.

15. A.M. ODLYZKO and H.J.J. te RIELE, Disproof of the Mertens conjecture, J. reine angew. Math. 357 (1985), 138-160. MR 86m:11070.

16. J.-M. FONTAINE, $I$ n'y a pas de variété abélienne sur $Z$, Invent. math. 81 (1985), 515-538. MR 87g:11073.

17. H. COHEN and J. MARTINET, Etude heuristique des groupes de classes des corps de nombres, J. reine angew. Math. 404 (1990), 39-76. 
18. A. BOREL and G. PRASAD, Finiteness theorems for discrete subgroups of bounded covolume in semi-simple groups, Publ: Math. I.H.E.S. 69 (1989), 119-171.

AT \& T Bell Laboraties

Murray Hill, New Jersey 07974

U.S.A. 